\title{
The Impact of Audiovisual Feedback on the Learning Outcomes of a Remote and Virtual Laboratory Class
}

\author{
Euan Lindsay and Malcolm Good, Member, IEEE
}

\begin{abstract}
Remote and virtual laboratory classes are an increasingly prevalent alternative to traditional hands-on laboratory experiences. One of the key issues with these modes of access is the provision of adequate audiovisual (AV) feedback to the user, which can be a complicated and resource-intensive challenge. This paper reports on a comparison of two studies of remote and virtual access to hardware, one with rich AV feedback and one without. The comparison shows that the learning outcomes of the remote access mode are dependent upon the richness of the feedback; the learning outcomes of the simulated access mode are largely robust to an absence of feedback. The students' preferences are affected by the feedback, with a clear preference for richer feedback.
\end{abstract}

Index Terms-Audiovisual (AV) feedback, comparative evaluation, learning outcomes, remote laboratories, student perceptions, virtual laboratories.

\section{INTRODUCTION}

$\mathbf{L}$ ABORATORY classes are widely accepted as a crucial part of an undergraduate engineering degree. Good pedagogical reasons, such as illustrating and validating analytical concepts, introducing students to professional practice and to the uncertainties involved in nonideal situations, developing skills with instrumentation, and developing social and teamwork skills in a technical environment [1]-[3], underlie the need for their inclusion in undergraduate curricula.

The traditional undergraduate lab class is comprised of a small group of students and a demonstrator, grouped around a piece of hardware located in a laboratory. The students conduct a series of experimental procedures as outlined in the laboratory handout, they record the data from the hardware, and they write up a report based on these data and the underlying theory in the week or two subsequent to the session.

This traditional, proximal model is coming under increasing pressure because of the changing demands of engineering courses. Scheduling increasingly large numbers of small groups of students, with each group requiring an hour (or more) of continuous and adequately supervised access to an expensive piece of laboratory equipment, is a difficult and expensive task. An increasingly prevalent solution to this dilemma is the use of alternative access modes-either simulation (or virtual) laboratories or remote access to real laboratory hardware.

Manuscript received August 12, 2007; revised September 01, 2008. First published May 26, 2009; current version published November 04, 2009.

E. Lindsay is with the Department of Mechanical Engineering, Curtin University of Technology, Perth, WA 6845, Australia (e-mail: e.lindsay@curtin. edu.au).

M. Good is with the Department of Mechanical Engineering, The University of Melbourne, Melbourne, VIC 3010, Australia.

Digital Object Identifier 10.1109/TE.2008.930510
Web-based remote labs have been offered by universities in undergraduate engineering courses since 1996 [4], with the number and sophistication of these efforts growing each year [5], [6].

An essential part of these remote and virtual laboratory classes is a technology-mediated interface through which the students control the equipment. Anecdotally, the transparency of this interface has been shown to be important in determining the effectiveness of the laboratory class [7], [8], and there are also strong theoretical indications that the interface should affect the learning outcomes [9]. The provision of this transparency can be expensive. Additional cameras and microphones add to the bandwidth requirements of a remote laboratory, and increasing the verisimilitude of a simulation requires greater computational power and/or more sophisticated models.

This paper addresses the extent to which this additional expense is justified. This paper reports on a comparison between a previously reported trial with audiovisual (AV) feedback [10] and another trial in which this feedback was absent. The analysis shows that the importance of the feedback differs between the remote and virtual modes. A range of laboratory outcomes are considered, including

- students' measured assessment outcomes;

- students' perceptions of their learning;

- students' engagement with the laboratory experience;

- students' preferred access modes.

\section{THE LABORATORY ClASS}

The laboratory which was investigated in this instance was the calibration of a piezoelectric accelerometer. This class forms a practical component for a third-year Mechanical Engineering unit in Data Acquisition and Control. In this laboratory experiment, the accelerometer is mounted on an electrodynamic shaker, which is excited using signals generated by a spectrum analyzer. The velocity of the accelerometer is also measured by a laser Doppler vibrometer. This velocity signal, and the accelerometer's own acceleration measurement, are analyzed using the spectrum analyzer. The hardware is shown in Figs. 1 and 2 .

This laboratory is conducted primarily through a single point of control, the spectrum analyzer (Fig. 3). As a result, the alternative access modes are simply a matter of providing a remote mechanism for controlling the spectrum analyzer, achieved in the remote implementation using a General Purpose Interface Bus (GPIB) connection.

A MATLAB Graphical User Interface (GUI) (shown in Fig. 4) was constructed to represent the spectrum analyzer and 


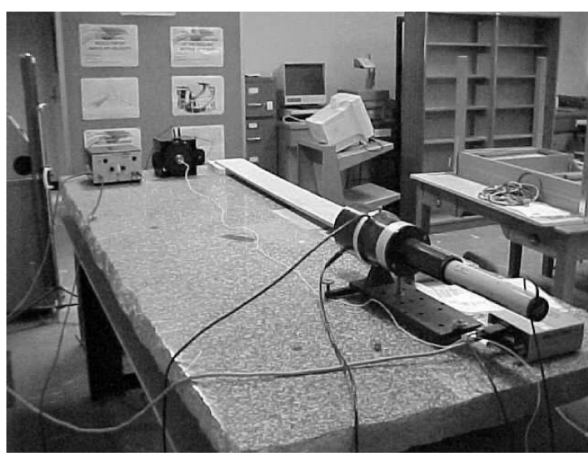

Fig. 1. The laboratory hardware.

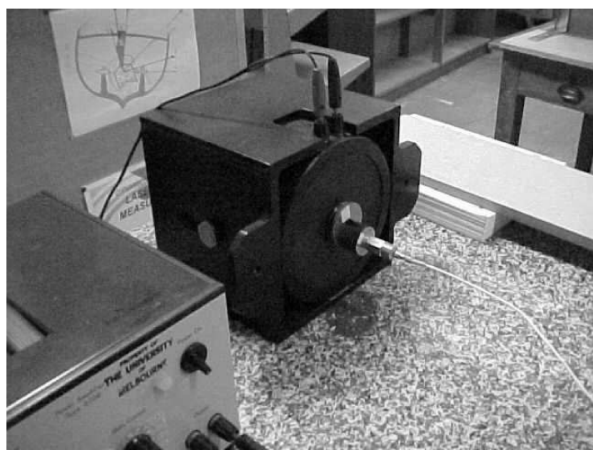

Fig. 2. The accelerometer mounted on the electrodynamic shaker.

to provide the user with access to the functionality of the spectrum analyzer that was necessary to perform this experiment.

A simulation of the system was also constructed, using the same GUI as the remote interface. This simulation used recorded data from the system to generate responses interactively for the user. The simulation access mode differed from the remote mode only in the students' belief as to whether there was actually real hardware involved or not. All other factors were kept the same. In this way some insight into the importance of the students' awareness of the access mode could be gained.

A microphone was placed in the laboratory, and Real Networks' Helix server was used to provide the students with an audio feed from the shaker. This audio feed allowed the students to hear the real equipment inside the laboratory, with essentially no time lag — as new commanded frequencies or signals were entered, the shaker would respond and the new frequency would be audible immediately. For the simulation version, the frequencies were generated by the simulation, and played through the computer speakers.

A Logitech webcam was used to provide students with a zoomed-in videostream of the shaker (Fig. 5). This videostream allowed the students to confirm that the shaker was not moving when it was silent, that it was visibly moving at low frequencies, and that it was blurred at higher frequencies. There was around a 1.5-s time lag between commands being sent through the GUI and the changed state of the shaker being visible on the screen.

For the simulation version, three prerecorded video streams were looped; one for each of the three distinct operating regimes (silent, low-frequency, high-frequency). The 1.5-s time lag was retained in the simulation to avoid introducing a potentially confounding difference between the modes.

The data from the cohort with AV feedback has previously been analyzed to determine the impact of mode upon these learning outcomes, and significant differences were found and reported [10]. This paper extends this analysis to consider the impact of not having rich AV feedback

\section{The ReseArch Protocol}

The student cohort for this laboratory class comprised third-year students drawn from a number of degree programs, including Mechanical, Mechatronic, and Environmental Engineering. The students had all completed a prerequisite course in linear feedback control (almost all in the semester prior to this course).

The trial was performed twice with consecutive cohorts, once with the AV feedback, and once without, instead providing just the experimental data through the spectrum analyzer GUI. All other aspects of the experience remained unchanged - the same laboratory handouts, the same interface, the same pictures used to orient the students in the remote and virtual modes. The trial with feedback included 146 students; the trial without feedback included 114.

The trials were designed to maximize the generalizability of the results [11]. All laboratory sessions were done in groups, at all times under the supervision of a laboratory demonstrator. Two laboratory demonstrators were used-one who supervised all of the groups in the first trial, and one who supervised all of the groups in the second trial. Students were randomly allocated to sessions in groups of six; however group sizes varied from four to seven based on students' attendance. Each group was randomly allocated to an access mode. Each student completed the laboratory only once, with the group (and corresponding access mode) to which they had been randomly allocated.

The laboratory class followed the same sequence for each of the three modes:

- a handout was provided to the students well before the laboratory;

- before the laboratory, the demonstrator took the group through a short (5-10 min) briefing ensuring they understood the theory of the laboratory;

- the group was oriented with the laboratory-in the proximal mode, this orientation was a "walk around" of the equipment; in the simulation and remote modes the orientation was a slideshow featuring photographs of the equipment (5 min);

- the group completed the laboratory, gathering their data ( $45 \mathrm{~min})$;

- students had two weeks from the date of their laboratory to submit their laboratory reports.

The laboratory handouts were the same for all students in both trials. There was no variation in the supplied materials for the different access modes. The prelaboratory briefing session was also the same for all students, although there were some inevitable variations depending on whether the students had questions throughout the briefing.

The physical layout of the laboratory was kept as similar as possible - the Remote and Simulation classes were held in the 


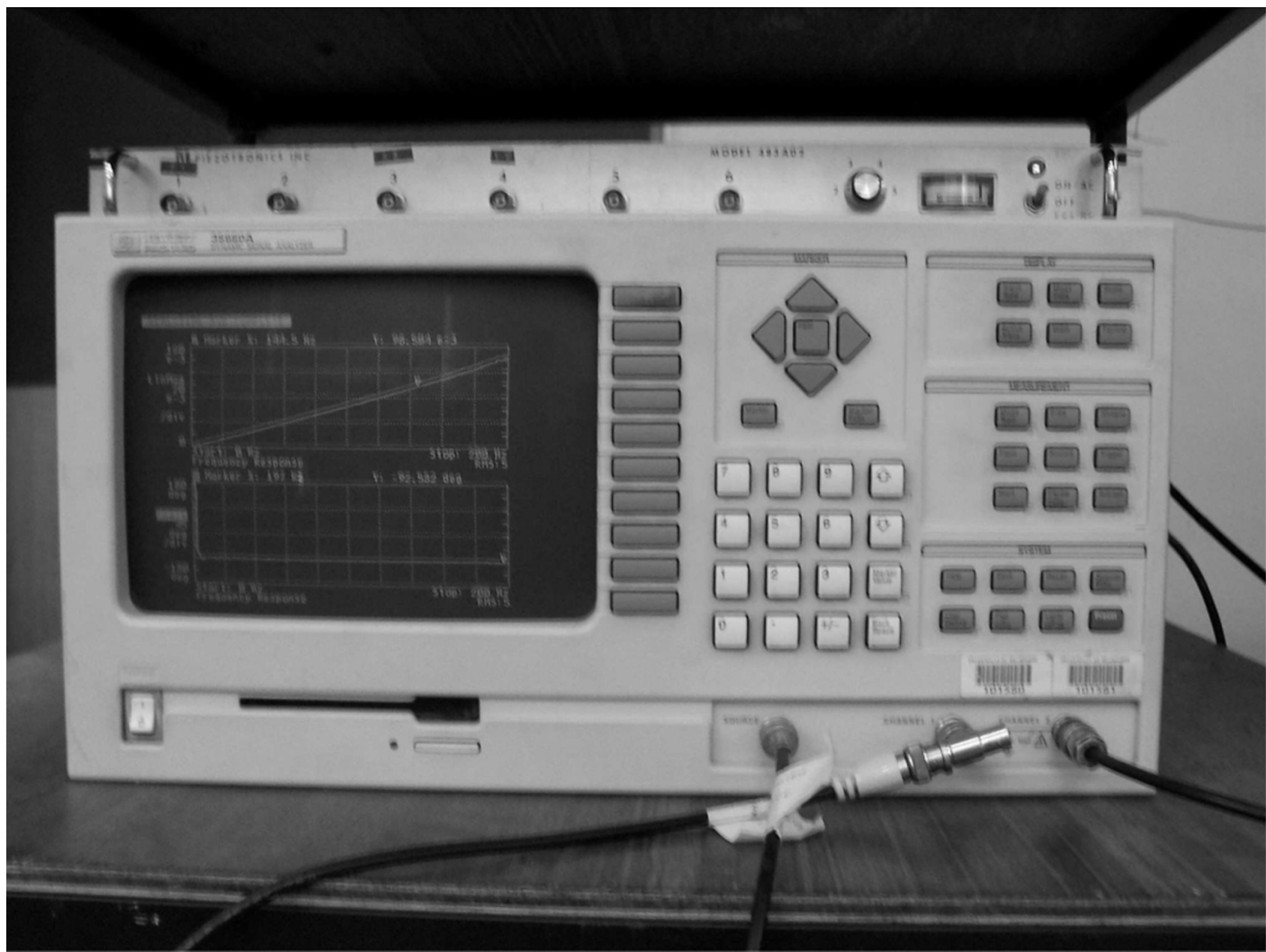

Fig. 3. Real analyzer interface.

same physical location, in a laboratory as similar as possible (albeit without real hardware) to the proximal mode.

The Assessment tools for each of the three modes were kept identical, with the same format for submissions, the same time allowed for submissions, and all submissions being marked by a single marker, who had also been the demonstrator for the laboratory class.

In addition to designing the trials to be as comparable as possible, a range of potentially confounding factors were also measured, so that they could be accounted for in the analysis

- the prior knowledge of the participants;

- participants' demographic information-Age, Gender, Subject (four separate units shared this same laboratory class), English Speaking Background/Non-English Speaking Background, Local Student/International Student, Full Time/Part Time, the Degree Program in which the Student is enrolled;

- preferred access modes/expectations;

- scheduling of the laboratory within the week, and within the semester.

Measuring these factors allowed for their contribution to the variance in results to be accounted for, and for the differences based on the access mode to be isolated and examined.

Students also completed a seven-question posttest to gage their perceptions of the experience. The questions were on a single page, and students were asked to complete the posttest after completing the laboratory work, but prior to completing the laboratory report. The following seven questions were asked.
1) These Laboratory Classes are being run with three access modes-Proximal (in person in the lab), Remote, and Simulation. What effect do you think your access mode had upon the laboratory class?

2) If given a free choice, which access mode would you have chosen and why?

3) Did you feel your calibration of the accelerometer was accurate?

4) What did you think the learning objectives of the laboratory class were?

5) What was the most important thing you learned from the laboratory class?

6) Did you find the laboratory class intellectually stimulating? Why/why not?

7) Any other comments, positive or negative?

Each of the students was allocated a Participant number, which was used as the primary key for referring to their responses. In this way their anonymity was preserved in accordance with the approval granted by the University's Research Ethics Committee.

\section{STUdENTS' LEARNING OUTCOMES}

The students' learning outcomes were measured through the assessment of their laboratory reports. The students each submitted a written report on their laboratory class, due two weeks after the completion of the laboratory. The reports were marked according to whether specific behaviors were represented. From these behaviors, 11 criteria marks were determined; and from 


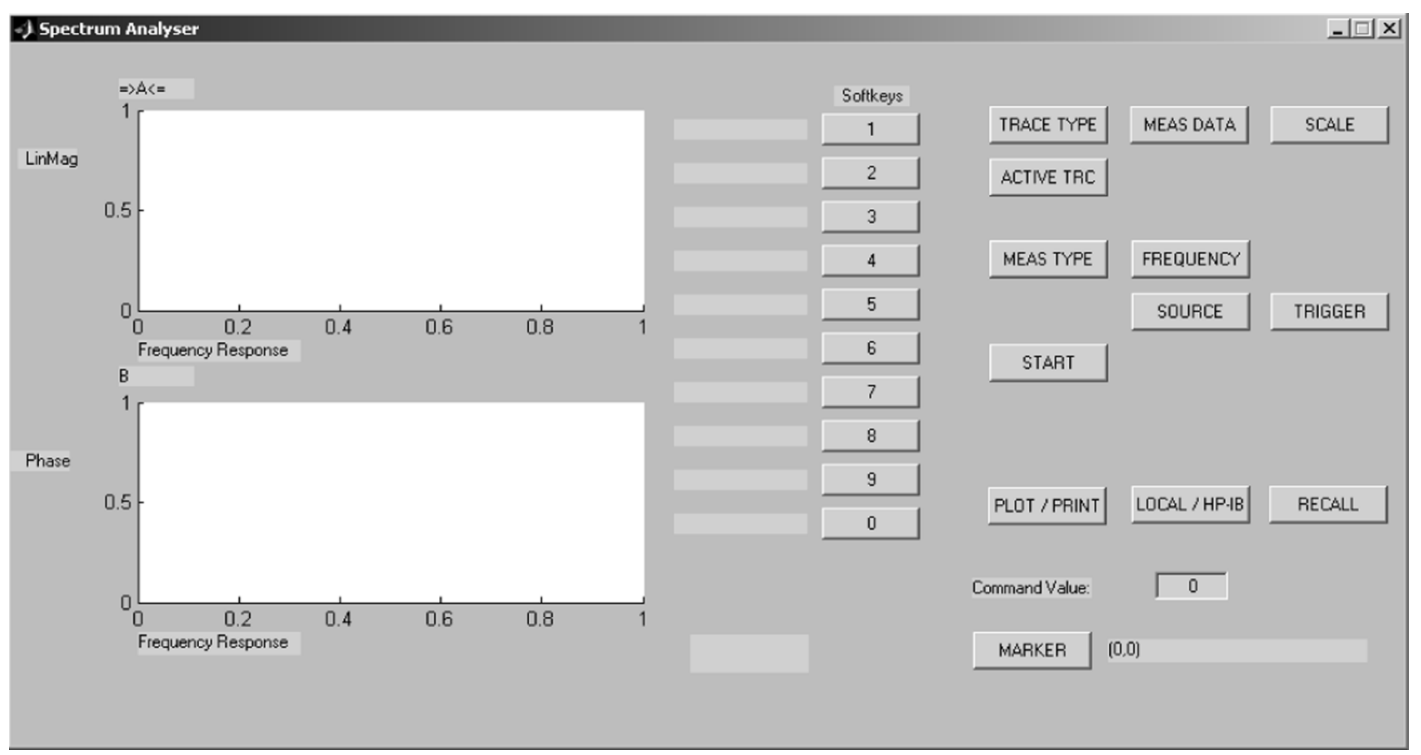

Fig. 4. GUI interface.

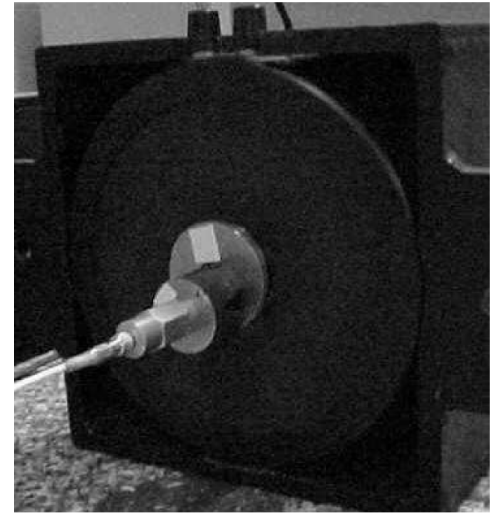

Fig. 5. Close-up image of shaker.

these 11 criteria marks, measures of eight learning outcomes were constructed. The interaction among the behaviors, criteria, and outcomes is illustrated in Fig. 6.

Each criterion has associated behaviors, varying from five to nine in number, depending upon the criterion. The eight outcomes are identified by letters A-H; the 11 criteria are numbered 1-11; and the behaviors within each criterion are referred to using Roman numerals.

The student's mark for a criterion is simply the number of associated behaviors displayed in his or her report. Attention is paid neither to which of the behaviors are included, nor where the behaviors appear in the report. For example, the behaviors for Criteria Four and Seven, the two criteria that contribute to the Exception Handling learning outcome, are as follows.

Criterion Four: Deviation from the "ideal" $H \omega$ versus $\omega$ straight line response. The actual response of the system will deviate from the "ideal" straight line response assumed in the laboratory handout. The student

I) identifies that the response deviates from the 'ideal' straight line;

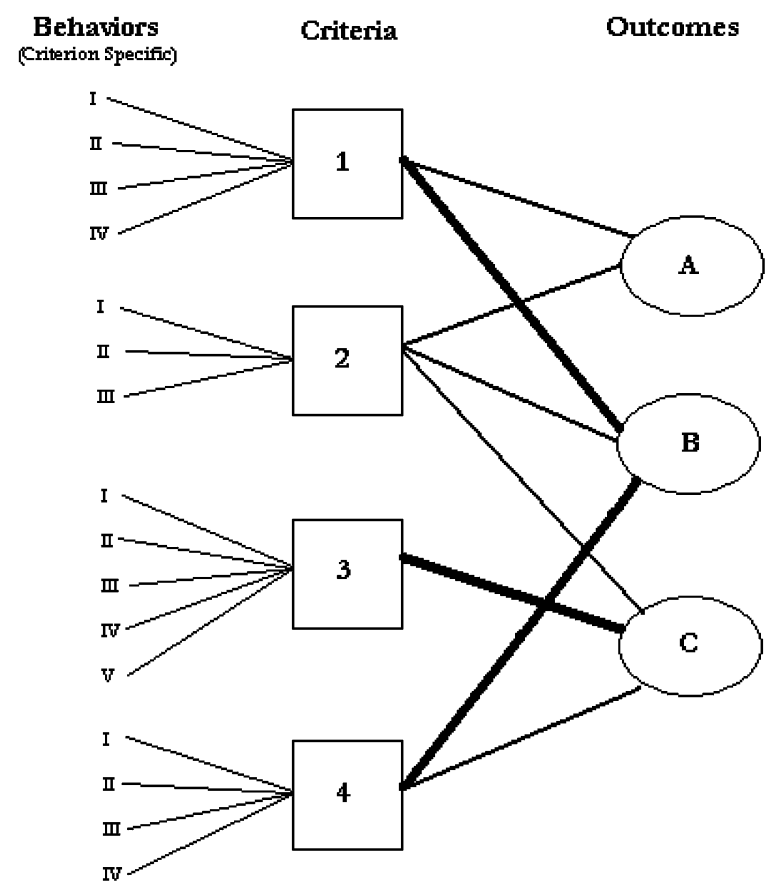

Fig. 6. Representation of the interaction between behaviors, criteria, and outcomes [10].

II) observes that the gradient of the line changes with frequency;

III) observes that response contains oscillation around the ideal straight line response;

IV) observes that the response does not have a zero magnitude at $\omega=0$;

V) provides explanations to explain this deviation; and

VI) identifies that this deviation compromises the calibration of the accelerometer as a sensor.

Criterion 7: Resonance/Antiresonance pair.

There is a resonance around the $200-\mathrm{Hz}$ frequency, and an antiresonance around the $220-\mathrm{Hz}$ frequency. The student 


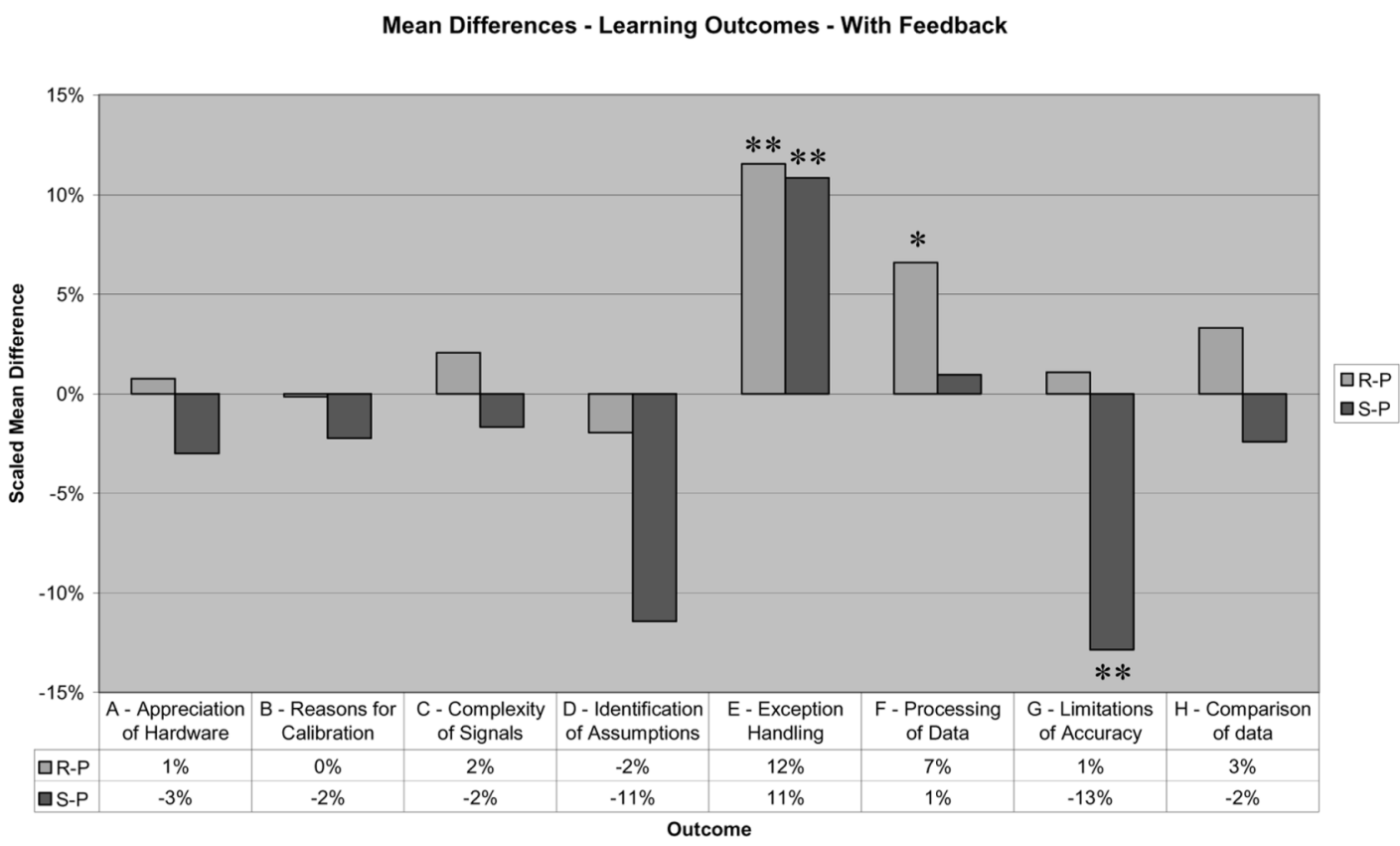

Fig. 7. Learning outcome differences—with feedback [10].

TABLE I

CRITERIA—OUTCOME LINKAGES

\begin{tabular}{|c|l|c|}
\hline No. & Criterion & Outcomes \\
\hline 1 & The relationship between $H(\omega)$ and $\omega$ & F \\
\hline 2 & The Calibration Constant $A$ (Final Value) & F,G \\
\hline 3 & The Calibration Process & C,F,H \\
\hline 4 & Deviation from the 'ideal' $H(\omega)$ vs $\omega$ straight line response & E,F \\
\hline 5 & Assumptions involved in simplifying the transfer function & D \\
\hline 6 & Linearity of the Accelerometer system & C,H \\
\hline 7 & Resonance / Anti-resonance pair & E \\
\hline 8 & The Piezoelectric Accelerometer & A \\
\hline 9 & The laser Doppler System & A \\
\hline 10 & Calibration as a process & B \\
\hline & & Spectral Analysis \\
\hline
\end{tabular}

I) identifies the existence of the resonance and the antiresonance;

II) indicates that these resonances compromise the calibration they have just completed;

III) notes the range of frequencies (180 to $230 \mathrm{~Hz}$ ) that are affected by the resonances;
IV) explains that the operating envelope for the accelerometer must not include the frequencies affected by the resonances;

V) indicates that these resonances will alter the gradient of the $|H(\omega)|$ versus $\omega$ curve;

VI) lists possible causes of these resonances;

VII) postulates possible remedies to correct these causes of resonance.

Thus, for example, a student who indicates that the response is not a straight line, and that it does not have a zero magnitude at $\omega=0$, but does not include any of the other behaviors, will score two for criterion four.

This fine-grained approach to marking the reports reduced the potential variation introduced by the marker. Marking therefore becomes a digital yes-no process rather than a continuous "feels like seventy percent" approach.

The lab class is intended to produce eight learning outcomes-three that are task-specific, and five that are generic skills usually associated with third-year engineering students.

Specific Outcomes:

A) appreciation of the hardware involved;

B) reasons for calibration;

C) the complexity of signals.

Generic Skills:

A) identification of assumptions;

B) exception handling;

C) processing of data;

D) limitations of accuracy;

E) comparison of data.

These outcomes are measured as linear combinations of the criteria marks. The links between criteria and their related outcomes are shown in Table I.

The bold letters indicate strong relationships. Strong relationships were weighted at 1.0 when determining the outcome score, 


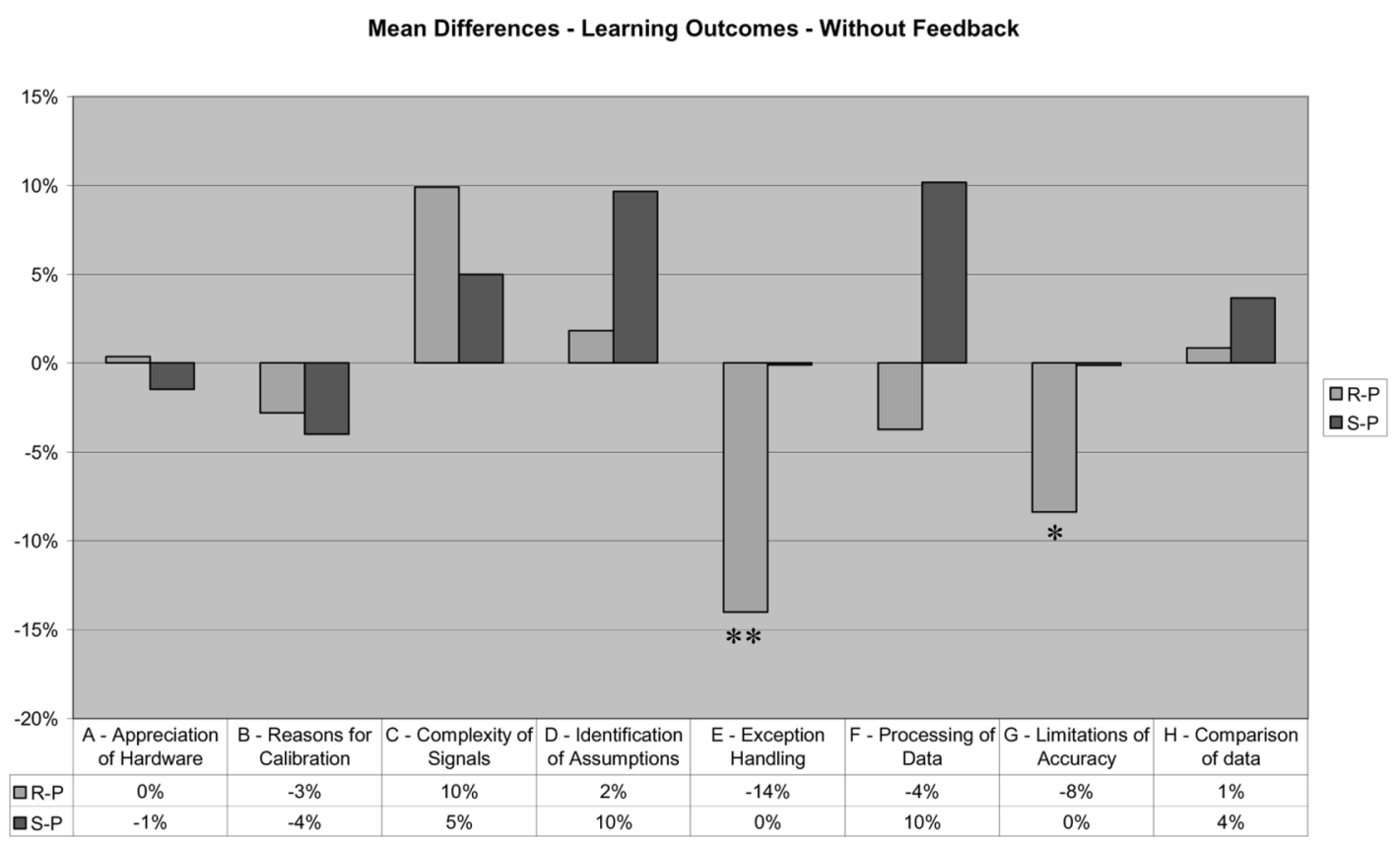

Fig. 8. Learning outcome differences-without feedback.

whereas weak relationships were weighted at 0.5 . Thus, the student's performance on Outcome E, Exception Handling, is the sum of their score on criterion seven plus half of their score on criterion four. From these relationships values for the eight different outcomes were determined for each student.

This marking scheme allowed for numerical measures of the students' learning outcomes to be obtained. This approach allowed for comparisons to be made between the three access modes. These comparisons of the students' learning outcomes show that the average performance varies depending upon which mode the students experienced (see Figs. 7 and 8).

The outcome-difference measures in Figs. 7 and 8 are scaled relative to the marks available for the outcome: they show the differences in learning outcomes between the traditional in-person mode $(\mathrm{P})$ and the remote $(\mathrm{R})$ or simulation $(\mathrm{S})$ alternative. Statistically significant differences (Mann-Whitney test) are indicated, thus, ${ }^{* *}(p<.01),{ }^{*}(p<.05)$. For example, on average with $\mathrm{AV}$ feedback present (Fig. 7), both the remote and simulation mode students dealt better with exceptions (Outcome E) than did those working in the proximal mode. In the absence of AV feedback (Fig. 8), however, the remote mode students performed less well than those in the proximal mode, whereas the simulation and proximal mode outcomes were about the same.

To investigate the impact of the AV feedback, the two datasets were normalized around their respective proximal group means, using the following formula:

$$
X_{\text {Normalized }}=\frac{\left(X_{\text {Raw }}-\text { Mean }_{\text {Pr oximal }}\right)}{\text { Mean }}
$$

The means of the distribution of the normalized values are shown in Table II. An independent samples t-test was used to determine whether the differences between the with-feedback group and the without-feedback group were statistically significant, with the significant differences marked with an asterisk.
For outcome E, Exception Handling, both modes showed a highly significant $(p=0.000)$ improvement in performance in the presence of AV feedback. This result clearly indicates that richer feedback assists the students in determining whether the data they are observing matches with their expectations.

For the simulation mode, there were four other outcomes for which there were significant differences (Table III), and in each case the without-feedback group outperformed the with-feedback group.

For outcomes D, F, G, and H, the simulation mode showed a significant degradation in performance in the presence of $\mathrm{AV}$ feedback. The learning outcomes of the remote mode, however, seem to be largely robust to the presence of $\mathrm{AV}$ feedback.

\section{A. The Impact of Feedback on Students' Outcomes}

Both trials found that the access mode has an impact upon the learning outcomes, but the impacts were substantially different for the two trials. The inclusion of AV feedback changes the nature of the differences between the different modes.

It is clear that the presence of AV feedback assists students in outcome E, Handling Exceptions, although the impact is greater for students in the remote mode. Whereas both remote and simulation mode students perform better than proximal mode students in the presence of feedback, students in the simulation mode do not perform worse than the proximal mode students in the absence of feedback.

With the exception of the handling of exceptions, the outcomes of students in the remote mode appear to be robust to the introduction or removal of AV feedback. They do perform better in the presence of feedback (particularly with regard to Outcome F, Processing Data, and Outcome G, Limitations of Accuracy), but these changes cannot be attributed (statistically at least) to the introduction of feedback. 
TABLE II

MEANS OF NORMALIZED DATA

\begin{tabular}{|c|c|c|c|c|c|c|c|c|}
\hline & Out_A & Out_B & Out_C & Out_D & Out_E & Out_F & Out_G & Out_H \\
\hline \multicolumn{9}{|c|}{ Proximal } \\
\hline With & 0.00 & 0.00 & 0.00 & 0.00 & 0.00 & 0.00 & 0.00 & 0.00 \\
\hline Without & 0.00 & 0.00 & 0.00 & 0.00 & 0.00 & 0.00 & 0.00 & 0.00 \\
\hline \multicolumn{9}{|c|}{ Remote } \\
\hline With & 0.05 & -0.02 & 0.09 & -0.04 & $0.66^{*}$ & 0.12 & 0.02 & 0.18 \\
\hline Without & 0.07 & -0.09 & 0.24 & 0.04 & $-0.26^{*}$ & -0.05 & -0.16 & 0.02 \\
\hline \multicolumn{9}{|c|}{ Simulation } \\
\hline With & -0.19 & -0.26 & -0.07 & $-0.21^{*}$ & $0.62^{*}$ & $0.02^{*}$ & $-0.30^{*}$ & $-0.11^{*}$ \\
\hline Without & -0.04 & -0.11 & 0.12 & $0.23^{*}$ & $0.00^{*}$ & $0.14^{*}$ & $0.00^{*}$ & $0.11^{*}$ \\
\hline
\end{tabular}

TABLE III

P-VALUES FOR T-TESTS-SIMULATION MODE

\begin{tabular}{|l|c|}
\hline \multicolumn{1}{|c|}{ Outcome } & $\mathrm{p}$ value \\
\hline D - Identification of Assumptions & .001 \\
\hline F - Processing of Data & .015 \\
\hline G - Limitations of Accuracy & .000 \\
\hline H - Comparison of data & .034 \\
\hline
\end{tabular}

Students in the simulation mode, however, demonstrate significant differences in their learning outcomes, with the surprising result that the presence of $\mathrm{AV}$ feedback serves to degrade their performance.

For outcome D, Identification of Assumptions, simulation mode students go from outperforming both other modes to underperforming both other modes. For outcome F, Processing of Data, outcome G, Limitations of Accuracy, and outcome H Comparison of Data, the simulation mode goes either from outperforming to equivalency, or equivalency to underperforming.

Despite providing the students with essentially the same laboratory experience, the two access modes provide significant differences in the outcomes-differences that can be attributed to the students' perceptions as to the reality of the hardware they are controlling.

\section{STUdents' Perceptions of Their LEARNing}

The students' perceptions of their learning were gauged through two of the posttest survey questions: "What did you think the learning objectives of the laboratory class were?" and "What was the most important thing you learned from the laboratory class?"

\section{A. Perceptions of Objectives}

In the trial with AV feedback, there were significant differences ( $p<0.05$ for a $3 \times 7$ contingency table) between the stu- dents' perceptions of the objectives of the laboratory in each mode (Fig. 9).

In the trial without $\mathrm{AV}$ feedback, there were also significant differences ( $p<0.05$ for a $3 \times 7$ contingency table) between the students' perceptions of the objectives of the laboratory in each mode (Fig. 10).

Both trials found that the access mode affected the students' perceptions of the objectives, with statistically significant differences between the modes for some of the categories of responses. The categories that displayed significant differences differed between the two trials. In both trials the percentage of responses in the hardware-related category were less prevalent in the Simulation mode. For the nonfeedback trial this difference was significant for both alternative modes, while for the feedback trial the difference was only significant for the remote-simulation comparison.

In the nonfeedback trial, students in the remote mode were significantly less likely to report Practical Link to Theory objectives than those in either of the alternative modes. In the feedback trial, however, Practical Link to Theory responses were slightly more prevalent in the remote mode than either of the two alternatives.

In the feedback trial, students in the Simulation mode were highly significantly more likely to display Calibration Principles category responses, whilst in the non-feedback trial there was no significant difference. The proportion of Calibration Principles responses for the simulation mode is almost unchanged between the two trials-it is a reduction in the overall number of Calibration Principles responses in the feedback trial that leads to this change.

In the feedback trial, the Proximal mode is significantly more likely to display Signal Analysis outcomes than either of the two nonproximal modes, whereas in the nonfeedback trial there is no difference. This result is due to the only group in either trial providing Signal Analysis responses being the Proximal mode in the feedback trial.

The impact of providing AV feedback to the students has been to shift the attention of the remote group back towards 


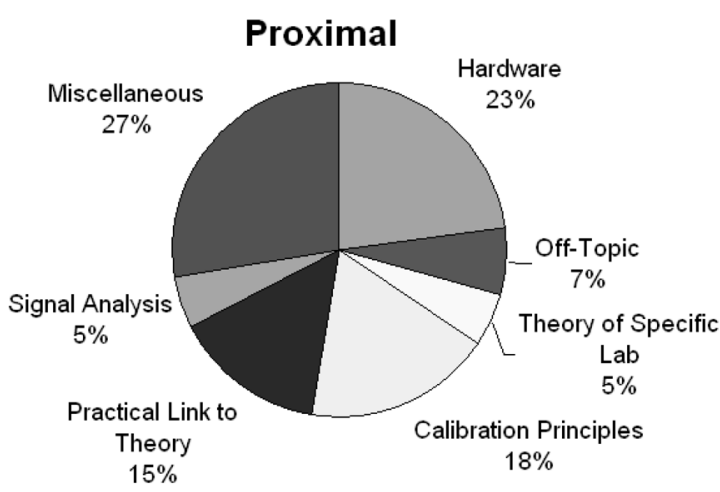

Simulation

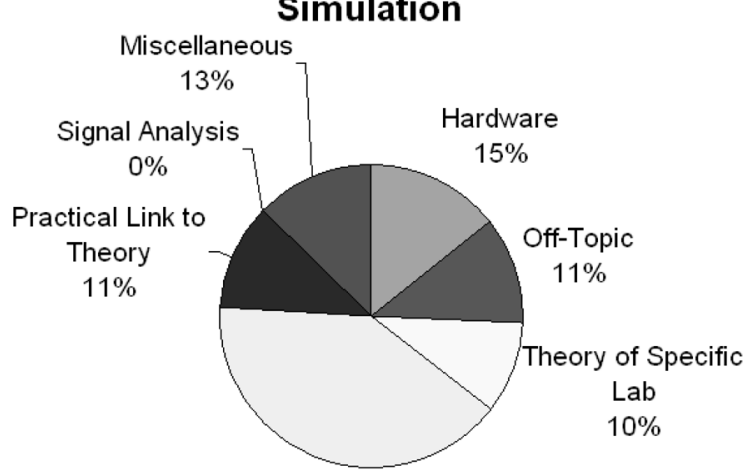

Calibration

Principles $40 \%$

Fig. 9. Students' perceptions of objectives — with feedback (adapted from [10]).
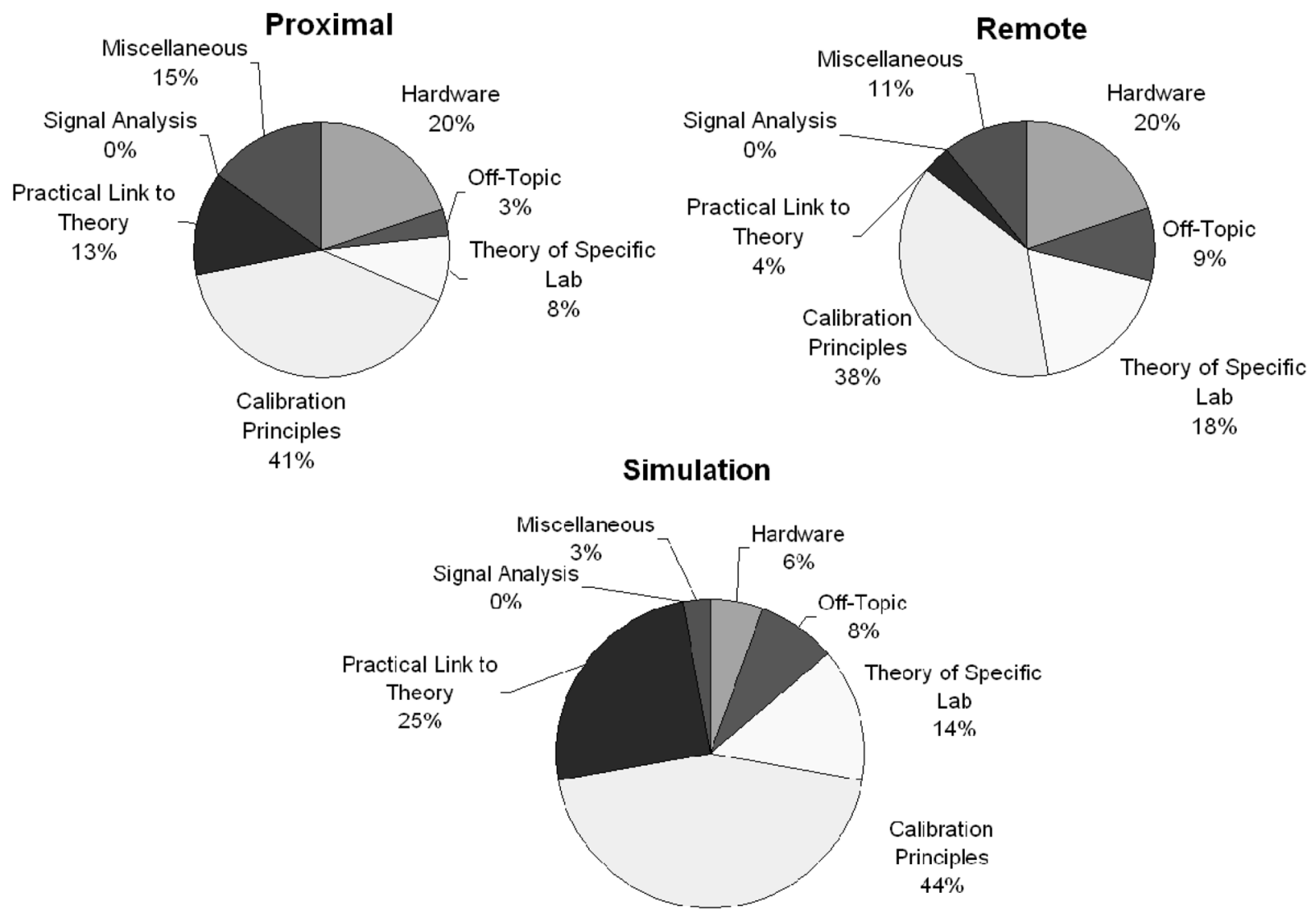

Fig. 10. Students' perceptions of objectives—without feedback. 

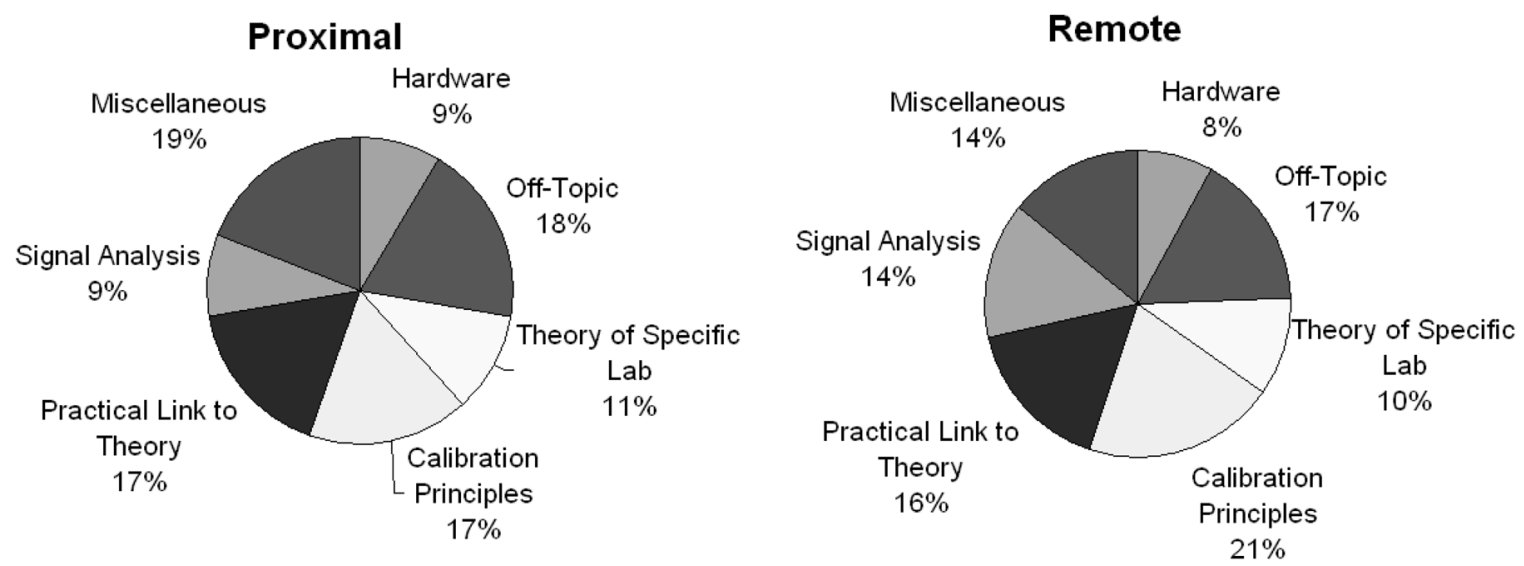

Simulation

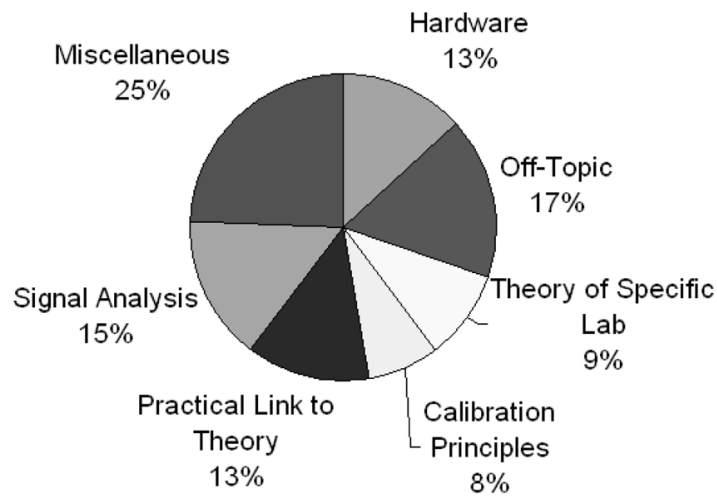

Fig. 11. Students' perceptions of outcomes—with feedback (adapted from [10]).

the Practical Link to Theory, and away from Calibration Principles, whereas the simulation group's perceptions of the objectives have remained largely unchanged.

\section{B. Perceptions of Outcomes}

In the trial with feedback, the students' perceptions of their learning outcomes remained largely unchanged across the three modes (Fig. 11).

Their perceptions of their outcomes also do not differ between modes for the trial without AV feedback (Fig. 12).

There are differences between the distributions in Figs. 11 and 12, however these differences are consistent across all three modes, suggesting that these differences are due to the change in trials, but not the interaction between these changes and the mode. Neither trial found any significant indication that the access mode affects the students' perceptions of the outcomes of the laboratories, which is in contrast to the changes shown in their perceptions of objectives.

\section{Perceptions of Objectives Versus Perceptions of Outcomes}

Both trials showed evidence of a mismatch between the students' perceptions of the laboratory objectives and of their learning outcomes. In the nonfeedback trial all three modes showed dissonances just short of the 0.05 level of significance, while in the feedback trial the remote and simulation modes showed clearly significant dissonance. This effect is most likely caused by differences in perceptions of objectives being matched with no differences in the perceptions of outcomes.
What students felt they learned from the laboratory is similar regardless of the mode or the trial, whereas their perceptions of what they were supposed to learn is affected by their mode-and this effect is greater when more authentic feedback is present.

\section{Students' Reported Levels of Engagement}

Students in the two trials reported substantially different levels of intellectual stimulation in the laboratories. The proportions of students who answered "yes" to the question "Did you find the laboratory intellectually stimulating?" varied little between the modes for each of the trials, but these proportions were much higher in the trial with the AV feedback (Fig. 13), than they were in the trial without (Fig. 14). Figs. 13 and 14 show that the proportions of "yes" and "no" answers did not vary across the modes, although there is a (nonstatistically significant) difference based upon the presence of AV feedback. Where there is a difference is in the reasons given by the students to justify their responses.

The responses suggest that students engage differently with the task on the basis of their access mode, but that the overall level of engagement appears unchanged - essentially, it seems that students find different things to get out of the experience, depending upon what is presented to them. Students in the proximal mode seemed to focus upon novelty-based motivations, stemming from their lack of prior similar experiences. Students in the remote mode appeared to focus upon the application of theory they had learned in lectures, with the laboratory engaging 


\section{Proximal}

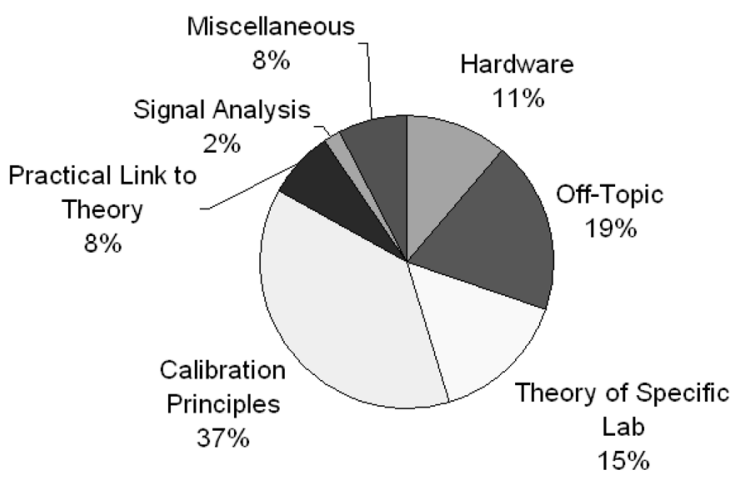

\section{Remote}

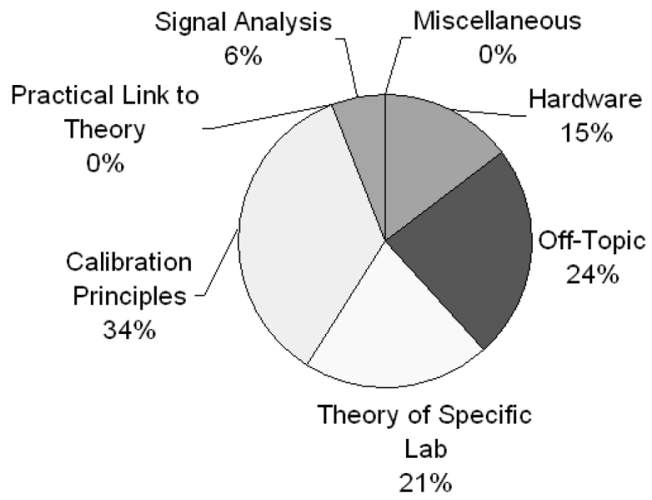

Simulation

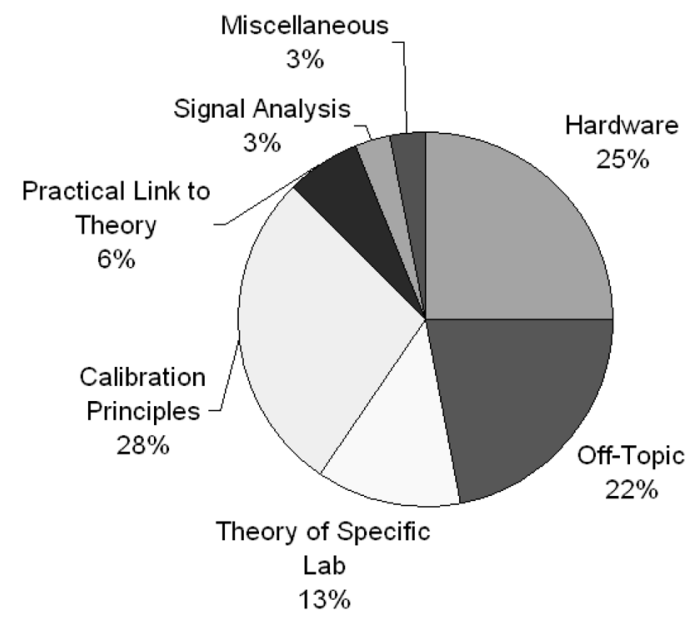

Fig. 12. Students' perceptions of outcomes—without feedback.
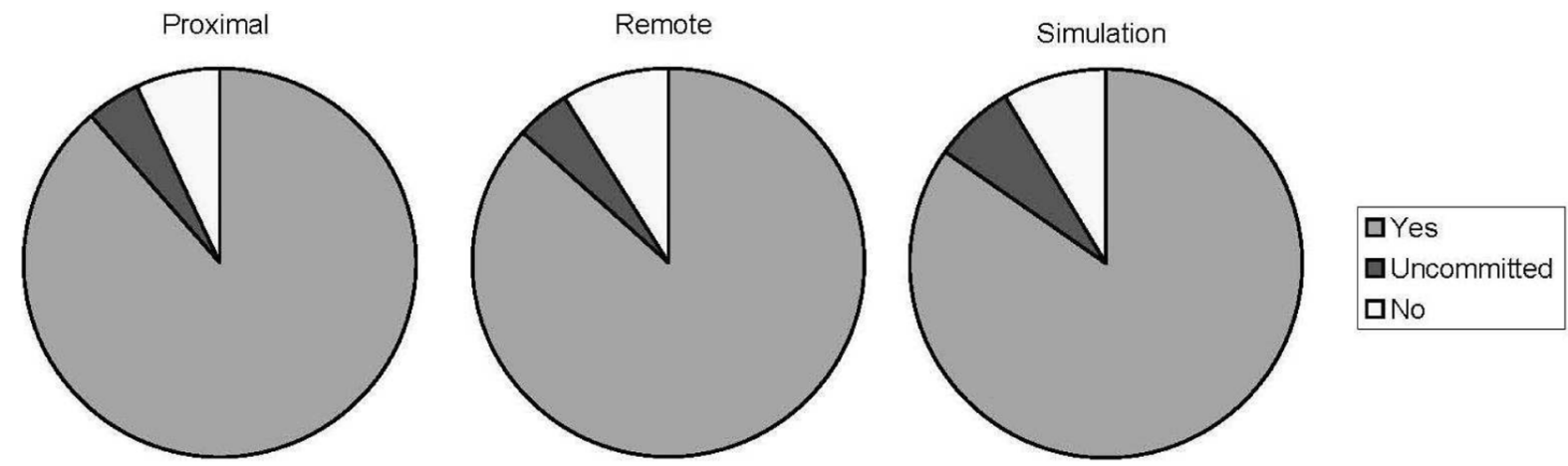

Fig. 13. "Did you find the laboratory intellectually stimulating?"-with feedback.

them as an opportunity to reinforce their theoretical knowledge. Students in the simulation mode most commonly focused upon their opportunity to learn in the laboratory, and more significantly, upon the process of learning — valuing their being made to think, and to answer questions.

Novelty and learning were both common reasons for answering yes in both trials. The inability to see the equipment was a reason for answering "no" in the nonfeedback trial that was-unsurprisingly-absent from the feedback trial. Much less prevalent in the feedback trial were responses on the theme "Not a real laboratory." While some students still expressed a preference for handling the real hardware, the presence of
AV feedback appeared to eliminate the resentment against the perceived artificiality of the experiment.

\section{E. Students' Preferred Access Mode}

Students were asked to respond to the question "If given a free choice, which access mode would you have chosen and why?" Their responses are tabulated in Table IV.

Table IV shows that there is a difference between the distributions for the two trials. Students in the nonfeedback trial were near-unanimous in their choice of the proximal mode; the students in the feedback trial - at least those that had undergone a nonproximal laboratory-were far more inclined to choose to 

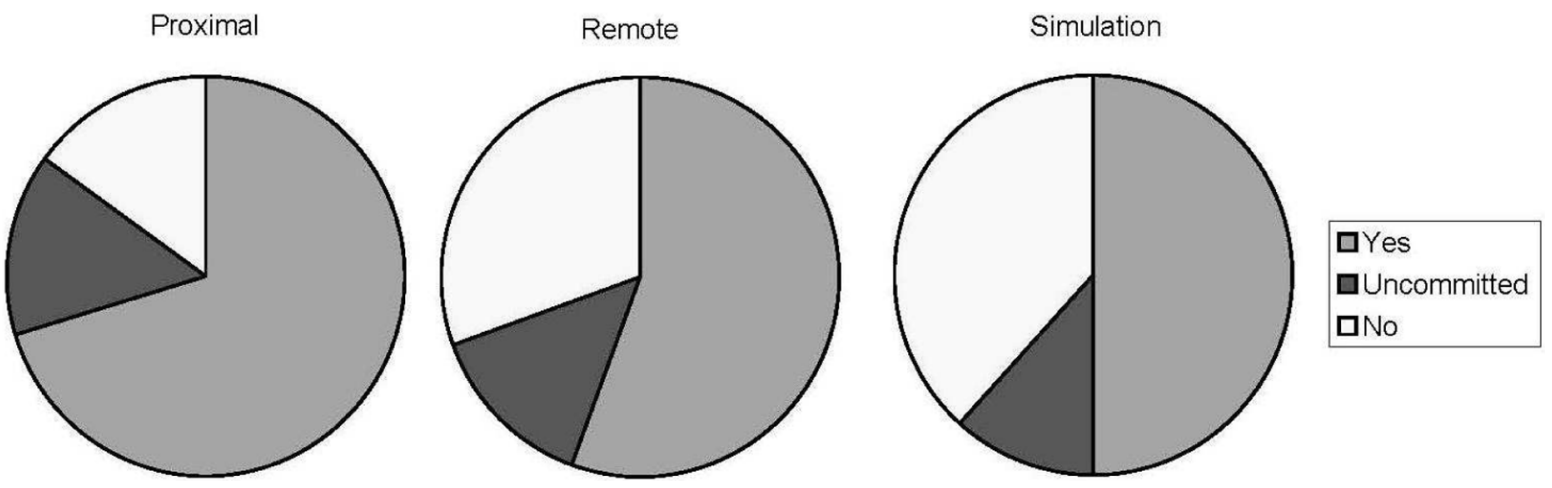

Fig. 14. "Did you find the laboratory intellectually stimulating?"-without feedback.

TABLE IV

StUdENTS' PREFERRED MODE Distributions (\%)

\begin{tabular}{|c|c|c|c|c|c|c|}
\hline & \multicolumn{3}{|c|}{ Non-feedback Trial } & \multicolumn{3}{c|}{ Feedback Trial } \\
\hline $\begin{array}{c}\text { Preferred } \\
\text { Mode }\end{array}$ & $\begin{array}{c}\text { Proxima } \\
\mathrm{I}\end{array}$ & $\begin{array}{c}\text { Remot } \\
\mathrm{e}\end{array}$ & $\begin{array}{c}\text { Simulatio } \\
\mathrm{n}\end{array}$ & $\begin{array}{c}\text { Proxima } \\
\mathrm{I}\end{array}$ & $\begin{array}{c}\text { Remot } \\
\mathrm{e}\end{array}$ & $\begin{array}{c}\text { Simulatio } \\
\mathrm{n}\end{array}$ \\
\hline Proximal & 89.8 & 81.1 & 95.2 & 86.7 & 66.3 & 59.1 \\
\hline Remote & 4.1 & 10.8 & 0.0 & 8.9 & 27.9 & 10.2 \\
\hline $\begin{array}{c}\text { Simulatio } \\
\mathrm{n}\end{array}$ & 6.1 & 8.1 & 4.8 & 4.4 & 5.8 & 30.7 \\
\hline
\end{tabular}

do so again. The reasons for the choices were mostly consistent between the trials - a preference for hands-on experience, and the need for visual feedback.

\section{F. The Impact of Feedback on Students' Perceptions}

The findings of the feedback trial clearly show that the students in all modes felt that the interface was adequate for the purposes of the laboratories - indeed there were some responses in the focus group interviews that the simulation interface may in fact have been superior to the unmediated proximal class [10].

This finding is in contrast to that for the nonfeedback trial, where around a third of the respondents in the remote mode indicated that the opacity of the interface was an issue, and that it needed to be improved-a finding that reinforces previous work in the field of remote laboratories. The simulation mode, which was working with an identical interface, did not find that the interface was an issue, suggesting that the mode is a more important factor than the interface. For the remote mode, the inclusion of the $\mathrm{AV}$ feedback serves to eliminate the complaints about the transparency of the interface.

The ability to directly see and hear what was going on was emphasized as a reason for the improvement in students' learning in many of the proximal mode responses for the feedback trial, and direct feedback was cited by many as a reason for their preference for the proximal mode of access. It appears that only in the simulation mode are the students' perceptions not affected by a lack of AV feedback.

This robustness to a lack of AV feedback is shown clearly when the students' perceptions of the learning objectives and outcomes of the laboratory are examined. What students felt they learned from the laboratory is similar regardless of the mode for each trial, whereas their perceptions of what they were supposed to learn is affected by their mode-and this effect is greater when more authentic feedback is present. Surprisingly, it is the simulation mode that remains stable throughout these changes, with the least variation in the distribution of responses between trials, suggesting that the feedback is less important in the simulation mode.

The students' perception of the learning outcomes and objectives may be linked in complex ways to their mode and the presence of feedback, but their engagement with the laboratory experience is linked in a much simpler way. Trials with feedback were reported as much more intellectually stimulating, with students also clearly preferring modes in which they could see and hear what was going on. While they are willing to acknowledge that they can learn without AV feedback, there is a clear message that they prefer not to.

\section{CONCLUSION}

A number of the dependent variables measured in this study displayed significant variation between the two trials. These variations indicate that the way in which students construct these outcomes is dependent upon the technology-mediated interface. Despite providing the students with essentially the same laboratory experience, the two access modes provide significant differences in the outcomes-differences that can be attributed to the students' perceptions as to the reality of the hardware they are controlling.

The presence or absence of AV feedback has a differing impact upon the two nonproximal modes. The remote mode displayed a substantial improvement in students' perceptions of outcomes with the introduction of AV feedback. The nonfeedback trial showed that students felt that the laboratory was artificial, and that the interface was not sufficiently transparent, and also led to inferior learning outcomes on a number of the criteria and outcomes that were measured. The trial which included AV feedback did not display these deficiencies-suggesting that the absence of the feedback is one cause for the poorer perceptions. Significant to note is that the students' measured learning outcomes were, in fact, largely robust to the presence or absence of the AV feedback.

The perceptions of students in the simulation mode seemed to be largely robust to the introduction of AV feedback, with only 
the students' reported levels of intellectual stimulation changing in response to the changed interface. This result suggests that the mental separation of not believing that there is real hardware at the other end of the interface is a stronger factor than the features of the interface when it comes to determining the students' opinions of the laboratory-if the students know that the equipment is not real, then they are less concerned about whether they are able to see it clearly. Whilst their attitudes did not substantially depend upon the presence or absence of AV feedback, there were significant degradations in their learning outcomes in the presence of the feedback, suggesting that the richer environment undermines their learning.

Aside from the presence or absence of AV feedback, the interfaces remain otherwise unchanged between trials. What is changed is the students' engagement with the laboratory experience-they way in which they choose to interact with the laboratory. The importance of the AV feedback-whether it is a necessity or a distraction-depends solely upon whether the students believe that the equipment is in fact real, or simply a simulation.

In the simulation mode, AV feedback has little impact upon their satisfaction, but can undermine their learning outcomes. In the remote mode, the $\mathrm{AV}$ feedback has a strong impact upon the students' satisfaction, but only a limited impact upon the learning outcomes.

This differing importance has significant consequences for the design of remote and virtual laboratory classes - the way in which the students engage changes their $\mathrm{AV}$ requirements, and laboratory designers must take these requirements into account. High-quality AV feedback is an important element of a good remote or virtual laboratory class-but the cost of not having that feedback varies between mode.

\section{REFERENCES}

[1] E. Scanlon, E. Morris, T. D. Paolo, and M. Cooper, "Contemporary approaches to learning science: Technology-mediated practical work," Stud. Sci. Educ., vol. 38, pp. 73-112, 2002.

[2] P. Antsaklis, T. Basar, R. deCarlo, N. H. McClamroch, M. W. Spong, and S. Yurkovich, "Report on the NSF/CSS workshop on new directions in control engineering education," IEEE Control Syst., vol. 19, pp. 53-58, Oct. 1999.

[3] L. D. Feisel and A. J. Rosa, "The role of the laboratory in undergraduate engineering education," J. Eng. Educ., vol. 94, pp. 121-130, Jan. 2005.

[4] B. Aktan, C. A. Bohus, L. A. Crowl, and M. H. Shor, "Distance learning applied to control engineering laboratories," IEEE Trans. Educ., vol. 39, no. 3, pp. 320-326, 1996
[5] J. Trevelyan, "Experience with remote laboratories in engineering education," in Proc. 14th Ann. Conf. Aust. Assoc. Eng. Educ., Melbourne, Australia, 2003, pp. 404-414.

[6] J. Ma and J. V. Nickerson, "Hands-On, simulated, and remote laboratories: A comparative literature review," ACM Comput. Surv., vol. 38, Sep. 2006.

[7] N. Givens and J. McShea, "Learning through remote practical experiments over the internet: a case study from teacher education," J. Inf. Technol. Teacher Educ., vol. 9, no. 1, pp. 125-135, 2000.

[8] R. Marin and P. J. Sanz, "The human-machine interaction through the UJI telerobotic training system," in Proc. ICEE2003, Valencia, Spain, 2003.

[9] E. D. Lindsay, M. C. Good, and S. Naidu, "A different kind of difference: Theoretical implications of using technology to overcome separation in remote laboratories," Int. J. Eng. Educ., vol. 23, no. 4, 2007.

[10] E. D. Lindsay and M. C. Good, "Effects of laboratory access modes upon learning outcomes," IEEE Trans. Educ., vol. 48, pp. 619-631, 2005.

[11] E. D. Lindsay, "Effects of laboratory access mode on learning outcomes," Ann. Res. Eng. Educ., vol. 2, no. 3, 2006.

Euan Lindsay received the Ph.D. degree in mechanical engineering from the University of Melbourne, Melbourne, Australia, in 2005.

$\mathrm{He}$ is a Senior Lecturer and the Discipline Leader for Mechatronic Engineering with Curtin University of Technology, Perth, Western Australia. His research interests include engineering education, telecontrol (particularly internet-based telecontrol), artificial neural networks, and rehabilitative technologies for people with sensing impairments.

Dr. Lindsay is a member of the Executive Committee of the Australasian Association for Engineering Education, and coedits the Australasian Journal of Engineering Education. He was the recipient of the 2007 Carrick Award for Australian University Teaching. In 2005, he was named one of the 30 Most Inspirational Young Engineers in Australia.

Malcolm C. Good (M'00) received the Ph.D. degree in mechanical engineering from the University of Melbourne, Melbourne, Australia, in 1975.

Previously, he was Program Leader for Integrated Manufacture in the Division of Manufacturing Technology of the Commonwealth Scientific and Industrial Research Organisation (CSIRO), Melbourne. From 1992 to 1996, he was Head of the Mechanical Engineering Department, University of Melbourne, where he is currently an Honorary Professorial Fellow. He has held visiting appointments with the Institute for Sound and Vibration Research (ISVR) of the University of Southampton, Southampton, U.K.; the Highway Safety Research Institute (HSRI) of the University of Michigan, Ann Arbor; General Electric Corporate Research and Development, Schenectady, NY; and Cambridge University, Cambridge, U.K. His research has been in the fields of fluid mechanics, vehicle and machine dynamics, highway geometrics, human factors of automobile and motorcycle control, vehicular impact with roadside structures, dynamics and control of machine tools and industrial robots, and, most recently, automotive drive-by-wire technologies.

Prof. Good has been President of the Australian Robot Association, Australian Contact Person for the International Advanced Robotics Program, and Interim Director of the Advanced Engineering Centre for Manufacturing. He is currently a Program Leader and Board Member of the Research Centre for Advanced $\mathrm{By}$-Wire Technologies. 